\title{
Equation of State Determination for Rhenium Using First-Principles Molecular Dynamics Calculations and High-Pressure Experiments
}

\author{
Shigeaki Ono \\ Research Institute for Marine Geodynamics, Japan Agency for Marine-Earth Science and Technology, 2-15 Natsushima-Cho, \\ Yokosuka, Kanagawa 237-0061, Japan \\ Correspondence should be addressed to Shigeaki Ono; sono@jamstec.go.jp
}

Received 10 August 2021; Accepted 21 January 2022; Published 18 February 2022

Academic Editor: Giuseppe Pellicane

Copyright (c) 2022 Shigeaki Ono. This is an open access article distributed under the Creative Commons Attribution License, which permits unrestricted use, distribution, and reproduction in any medium, provided the original work is properly cited.

The room-temperature bulk modulus of rhenium (Re) was measured in the pressure range 0 to $115 \mathrm{GPa}$ using a laser-annealing diamond anvil cell and the synchrotron X-ray diffraction method. Thermal properties of Re were investigated up to $4000 \mathrm{~K}$ based on first-principles molecular dynamics calculations, and the equation of state for Re was determined using experimental and calculated data. A Vinet equation of state fitted to the $300 \mathrm{~K}$ data yielded a bulk modulus of $K_{T 0}=384 \mathrm{GPa}$ and a pressure derivative of $K_{T 0}^{\prime}=3.26$. The contribution of thermal pressure was determined to have the form $\Delta P_{t h}=\left[\alpha K_{T}\left(V_{a}\right)+\left(\partial K_{T} / z T\right)_{V} \ln \left(V_{a} / V\right)\right] \Delta T$. When $\alpha K_{T}\left(V_{a}\right)$ was assumed to be constant, the fit to the data yielded $\alpha K_{T}\left(V_{a}\right)=0.0056 \mathrm{GPa} / \mathrm{K}$. In contrast, the volume dependence of the thermal pressure was very small, and fitting yielded a value of $\left(\partial K_{T} / z T\right)_{V}=-0.00042$.

\section{Introduction}

Rhenium ( $\mathrm{Re})$ is a group VII transition metal that crystallizes in a hexagonal close-packed (hcp) structure and has an axial $c / a$ ratio of 1.615 , slightly less than the ideal hcp value of 1.633. The space group is $P 63 / \mathrm{mmc}$, and two Re atoms occupy the 2 c Wyckoff sites $(1 / 3,2 / 3,1 / 2)$ and $(2 / 3,1 / 3,3 / 4)$. The high-pressure behaviors of Re have been studied widely as Re has a high bulk modulus value $(\sim 350 \mathrm{GPa})$ compared with other metals. Bridgman [1] reported that Re was the least compressible of all the metals, but it is now known that Re has the second-highest bulk modulus value after Os. An X-ray diffraction study carried out by Liu et al. [2] reported that the bulk modulus was $336 \mathrm{GPa}$ at room temperature. Recently, higher values of $\sim 353 \mathrm{GPa}$ have been reported by Dubrovinsky et al. [3] and Anzellini et al. [4], obtained using synchrotron X-ray diffraction at room temperature. Ultrasonic measurements by Manghnani and Katahara [5] gave a value of $360 \mathrm{GPa}$, which was in agreement with those reported in recent X-ray diffraction experiments. In contrast, first-principles calculations have shown discrepancies for the bulk modulus at $0 \mathrm{~K}$. Fast et al. [6] reported a value of $447 \mathrm{GPa}$, obtained by means of local-density approximation (LDA). Steinle-Neumann et al. [7] calculated values of $382 \mathrm{GPa}$ using LDA and $344 \mathrm{GPa}$ using the generalized gradient approximation (GGA). The LDA and GGA values calculated by Lv et al. [8] were 389 and $376 \mathrm{GPa}$, respectively.

Since Re is used as gasket material in diamond anvil cell experiments, there is considerable interest in its highpressure behavior. Recently, the double-stage and the toroidal diamond anvil cells have extended the pressure range over $600 \mathrm{GPa}$ [3, 9-11]. In such experiments, the equation of state (EOS) for Re has often been used as a pressure marker at extremely high pressures. An investigation of EOS could therefore improve the reliability of high-pressure experimental studies. Reliable data for high temperatures are still not available as the uncertainty in the temperature is nonnegligible in high-pressure experiments. Recently, 
theoretical studies using first-principles calculations have investigated the physical properties of materials at high pressures and temperatures. Although it is known that firstprinciples molecular dynamics calculations have significant advantages in terms of investigating the physical properties of materials at high temperatures, first-principles molecular dynamics calculations have been rarely used as they require huge computational resources.

We used density functional theory to investigate the thermal properties of Re and performed high-pressure experiments to determine the room temperature EOS for Re. This combination of first-principles molecular dynamics calculations and high-pressure experiments allowed us to determine reliable values for the thermoelastic properties over a wide range of pressures and temperatures.

\section{Methods}

First-principles calculations were performed using the Vienna Ab Initio Simulation Package (VASP) [12]. The PBEsol functional for the exchange-correlation potential was used in the GGA calculations [13]. The electronic wave functions were expanded using a plane-wave basis set with a cutoff energy of $600 \mathrm{eV}$, and the electron-ion interactions were described using the projector augmented wave (PAW) method. The PAW potential for Re had an outermost cutoff radius for the valence orbital of $1.434 \AA$, with a valence configuration of $5 \mathrm{~d}^{5} 6 \mathrm{~s}^{2}$. We used a 54-atom supercell with gamma-point Brillouin zone sampling and a time step of $1 \mathrm{fs}$ for first-principles molecular dynamics simulation at constant volume. Simulations were run using a constant NVT ensemble (i.e., conservation of numerical quantity, volume, and temperature) with the Nosé [14] thermostat for 5-10 ps after equilibration. A total of 37 different pressure-volume conditions were taken into consideration in the calculations. The pressure and temperature ranges were $0-379 \mathrm{GPa}$ and $300-4000 \mathrm{~K}$, respectively, and the thermal pressure was calculated for each volume.

High-pressure X-ray diffraction experiments were carried out using a diamond anvil cell (DAC) with a laserannealing system. The culet size of the diamond anvil was 300 or $150 \mu \mathrm{m}$, and Re reagent powder (99.9\% purity) was used as the starting material. Powdered sample was sandwiched between pellets of $\mathrm{NaCl}$ powder, which was used as the pressure-transmitting medium and the pressure reference. The sample was loaded into a hole $50-100 \mu \mathrm{m}$ in diameter drilled into a Re gasket, which was pre-indented to a thickness of $30-50 \mu \mathrm{m}$. The starting material was compressed at room temperature using a symmetrical or motordriven DAC $[15,16]$. An angle-dispersive X-ray diffraction method was used to investigate the sample on two synchrotron beamlines: AR-NE1A at the Photon Factory and BL10XU at SPring-8. Experimental assemblies for these synchrotron X-ray measurements have been described elsewhere $[17,18]$. The wavelength of the monochromatic incident X-ray beam was $\sim 0.41 \AA$, and powdered X-ray diffraction patterns were obtained on an image plate system (Rigaku R-AXIS, Japan). Spectra of the sample were collected over 1-10 min to determine the cell parameters and the volume of $\mathrm{Re}$, and observed intensities on the imaging plates were integrated as a function of $2 \theta$ to obtain conventional, one-dimensional diffraction profiles. Sample pressure was calculated from the $\mathrm{NaCl}$ unit cell volume, using the EOS for $\mathrm{NaCl}$ developed by Dorogokupets and Dewaele [19]. EOS parameters for Re were obtained from a least-squares fit to the pressure-volume data of the Vinet EOS [20]. The sample was compressed to the desired pressure at room temperature, which was confirmed by the pressure scale of the Raman spectra from the diamond [21]. Since the differential stress during room temperature compression causes a significant systematic bias in the relationship between the pressure and structural properties, the samples were heated after each change in pressure using an infrared laser, to reduce any differential stress in the sample.

The total pressure under high-temperature and high-pressure conditions was estimated from the roomtemperature EOS obtained from experimental data and the thermal pressure from the first-principles molecular dynamics calculations. The pressures of the solids can be expressed as

$$
P(V, T)=P_{s t}(V, 300)+P_{t h}(V, T),
$$

where $P(V, T)$ is the total pressure $P$ at volume $V$ and temperature $T$. The first and second terms on the right-hand side represent the relationship between pressure and volume at $300 \mathrm{~K}$ and the thermal pressure at volume $V$, respectively. In this case, the Vinet EOS [20] was used for the first term in equation (1).

$$
\begin{aligned}
P_{s t}(V, 300)= & 3 K_{T 0} \frac{\left[1-\left(V / V_{0}\right)^{(1 / 3)}\right]}{\left(V / V_{0}\right)^{(2 / 3)}} \\
& \cdot \exp \left\{\frac{3}{2}\left(K_{T 0}^{\prime}-1\right)\left[1-\left(\frac{V}{V_{0}}\right)^{(1 / 3)}\right]\right\},
\end{aligned}
$$

where $K_{T 0}$ is the isothermal bulk modulus and $K_{T 0}^{\prime}$ is $\left(\partial K_{T} / \partial P\right)_{T}$ at ambient temperature. In the thermal pressure EOS [22], $P_{t h}$ can be written as follows:

$$
\begin{aligned}
P_{t h}(V, T)= & \alpha_{0} K_{T 0}\left(T-T_{300}\right)+\left(\frac{\partial K_{T}}{\partial T}\right)_{V}\left(T-T_{300}\right) \\
& \cdot \ln \left(\frac{V_{0}}{V}\right)+\left(\frac{\partial^{2} P}{\partial T^{2}}\right)_{V}\left(T-T_{300}\right)^{2},
\end{aligned}
$$

where $\alpha_{0},\left(\partial K_{T 0} / \partial T\right)_{V}$, and $\left(\partial^{2} P / \partial T^{2}\right)_{V}$ are the coefficient of the volume thermal expansion at ambient condition, the temperature derivative of the isothermal bulk modulus at constant volume, and the second temperature derivative of the pressure at constant volume, respectively. Finally, equation (1) is expressed as 


$$
\begin{aligned}
P(V, T)= & 3 K_{T 0} \frac{\left[1-\left(V / V_{0}\right)^{(1 / 3)}\right]}{\left(V / V_{0}\right)^{(2 / 3)}} \exp \left\{\frac{3}{2}\left(K_{T 0}^{\prime}-1\right)\left[1-\left(\frac{V}{V_{0}}\right)^{(1 / 3)}\right]\right\} \\
& +\left[\alpha_{0} K_{T 0}\left(V_{0}\right)+\left(\frac{\partial K_{T 0}}{\partial T}\right)_{V} \ln \left(\frac{V_{0}}{V}\right)+\left(\frac{\partial^{2} P}{\partial T^{2}}\right)_{V}(T-300)\right](T-300) .
\end{aligned}
$$

Equation (4) was used to fit the pressure-volumetemperature data from our experiments and calculations.

\section{Results}

The room-pressure unit-cell parameters of the starting material were $a=2.7620(1), \quad c=4.4592(2) \AA$, and $V=29.460(2) \AA^{3}$, values that are in good agreement with those from previous studies in the literature. Typical diffraction data are shown in Figure 1. After compression to the desired pressure, stress broadening of each diffraction peak was observed (Figure 1(a)), which decreased drastically after laser annealing (Figure 1(b)). This indicates that the differential stress under compression was released on annealing. Diffraction data after annealing were used to determine the EOS since the differential stress often causes bias in the relationship between volume and pressure. Figure 2 shows the changes in the volume and lattice parameters as the pressure increased. The volumes and pressure data were fitted to the Vinet EOS [20] using the least-squares method, yielding values of $V_{0}=8.878(12)$ $\mathrm{cm}^{3} / \mathrm{mol}, K_{T 0}=383(13) \mathrm{GPa}$, and $K_{T 0}^{\prime}=3.26(30)$ (Table 1). The value of the bulk modulus obtained in our experiments differs slightly from those reported from previous experiments without annealing (Table 2). Figure 2 also shows the comparison of volume and lattice parameters between experimental and calculated results. The calculated lattice parameters of $a$-axis are in good agreement with the experimental values. In contrast, small discrepancies for $c$-axis and volume are confirmed between the calculated and experimental values. This indicates that the experiments are advantageous for the EOS study at room temperature.

First-principles molecular dynamics calculations were carried out at $0-428 \mathrm{GPa}$ and $300-4000 \mathrm{~K}$ (Figure 3 ). A total of 35 volume-pressure-temperature data points for hcp Re were used to analyze the EOS. Figure 4 shows the volume dependence of thermal pressures. At low temperatures $(<1000 \mathrm{~K})$, the dependence of thermal pressure on volume was small, but the volume dependence increased with temperature. The significant dependence of thermal pressure on temperature indicates that the Grüneisen parameter has a large temperature dependence at higher temperatures $(>2000 \mathrm{~K})$. Similar behavior has been reported for metals, ionic crystals, and rare gas solids [23-25]. Figure 5 shows the fitted isothermal compressibility curves at 300, 2000, 4000, and $8000 \mathrm{~K}$. As the pressure increases, the dependence of the pressure on the thermal expansion decreases: this result is in good agreement with the typical properties of condensed materials.

\section{Discussion}

The bulk modulus for Re and its derivative have been determined previously through experiments and calculations (Table 2), and the discrepancies in the calculated value are larger than those for the experimental values. In the case of calculations, the differences in the approximations used in the first-principles method have led to different values for the elastic properties since the uncertainty in the approximation is nonnegligible. It is known that the value of the bulk modulus obtained using LDA is higher than that obtained from GGA. According to previous studies, calculated values for the bulk modulus have a tendency to be higher than experimental values.

Our experimental value for the bulk modulus of $383 \mathrm{GPa}$ is slightly higher than those reported in previous experimental studies (Table 2). The difference between our experimental method and the approaches used in previous studies is related to the use of annealing before the acquisition of X-ray diffraction data. It is known that differential stress accumulates in the sample chamber during room-temperature compression, and this stress often has an influence on the measurement of the elastic properties of solids. In our study, laser-heated annealing was performed to minimize the influence of the differential stress at each pressure increment. The differences between our value and those reported in previous studies are therefore likely to be due to the differential stress in the sample chamber.

Vohra et al. [26] reported a variation in the axial ratio $(c / a)$ with the pressure of up to $216 \mathrm{GPa}$. The value of the c/a ratio is remarkably constant, with values of 1.615 and 1.614 at 0 and $216 \mathrm{GPa}$, respectively. In contrast, recent experimental studies $[3,4]$ have shown a reduction in the $c / a$ ratio with increasing pressure. Our experimental results indicated a reduction in the $c / a$ ratio of about $0.2 \%$ up to $120 \mathrm{GPa}$, which was in general agreement with the value reported by Anzellini et al. [4]. A reduction in the $c / a$ ratio has also been reported for other hcp metals, such as osmium, beryllium, cobalt, samarium, and dysprosium [27-30]. Theoretical investigations $[31,32]$ have predicted a minimum in the $c / a$ ratio at very high pressures $\left(V / V_{0} 0.75\right)$; however, no evidence for this minimum $c / a$ ratio was observed in our experimental study. This discrepancy might be due to the differences in the pressure conditions between our experiments and previous theoretical calculations. We investigated the variation in the $c / a$ ratio at pressures corresponding to $V / V_{0}>0.8$. To assess this interesting behavior of structure, it will be necessary to perform experiments at higher pressures in future work. 


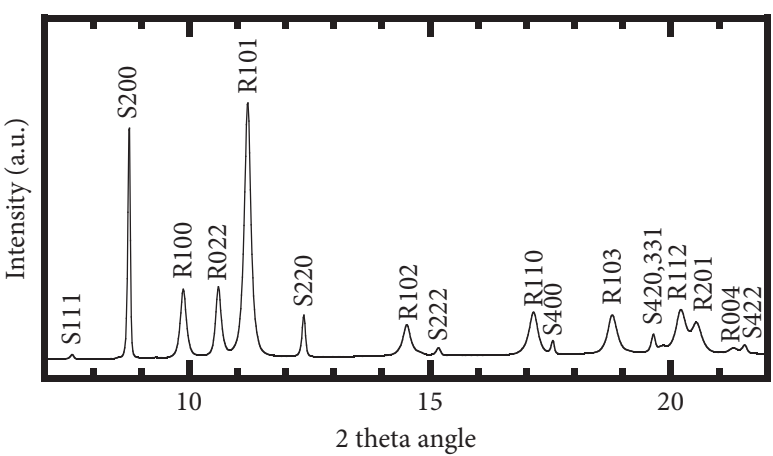

(a)

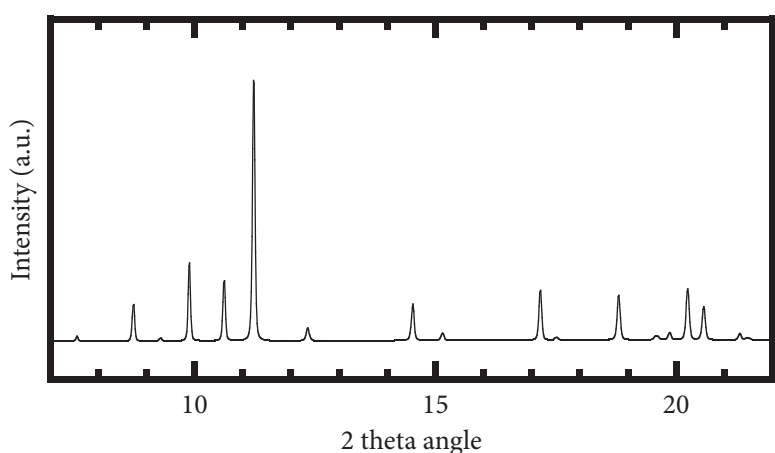

(b)

Figure 1: Comparison of powder X-ray diffraction data, before and after annealing. The upper and lower data show the results before and after annealing, respectively. Labels for the diffraction peaks are as follows: R-Re with hexagonal structure; S-B1-type NaCl. Numbers on the labels correspond to the indices of hexagonal or cubic symmetry. Monochromatic incident X-ray beam wavelength: $\lambda=0.4110 \AA$.
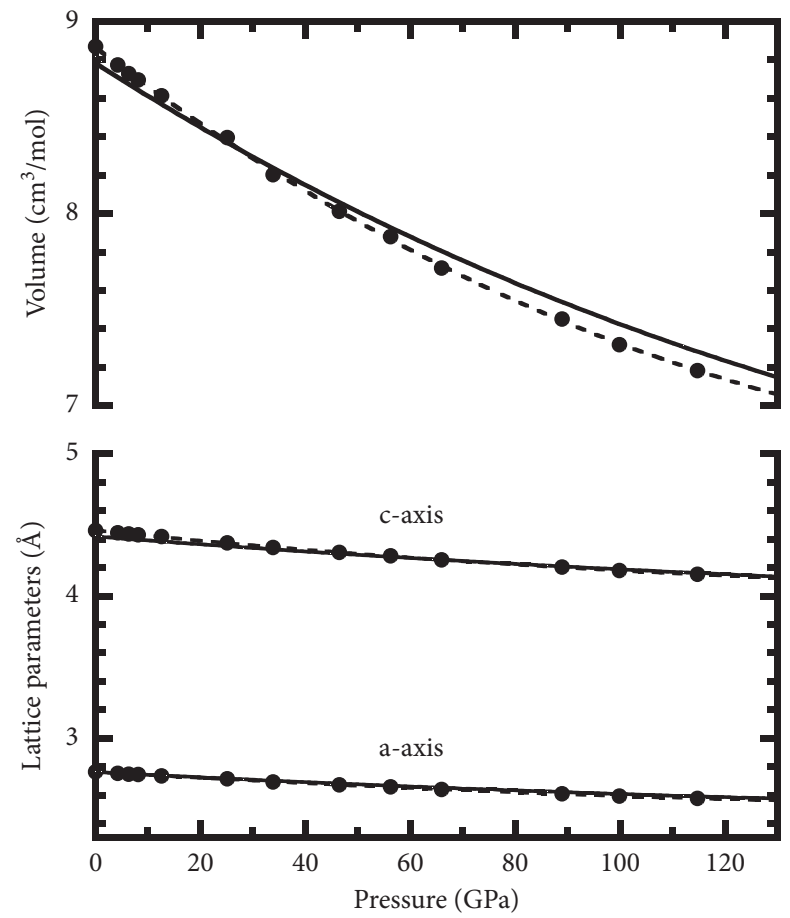

Figure 2: Experimental data on lattice parameters and volume of Re at $300 \mathrm{~K}$. Circles represent the lattice parameters and unit cell volumes of Re with a hexagonal structure, obtained from high-pressure experiments. Dashed lines show the fitted curves. Solid lines show the calculated lattice parameters and unit cell volumes at $300 \mathrm{~K}$.

TABLE 1: Thermoelastic parameters of Re.

\begin{tabular}{lc}
\hline Parameter & \\
\hline Experiment & $8.878(12)$ \\
$V_{0}\left(\mathrm{~cm}^{3} / \mathrm{mol}\right)$ & $383(13)$ \\
$K_{T O}(\mathrm{GPa})$ & $3.26(30)$ \\
$K_{T 0}^{\prime}$ & \\
First-principles molecular dynamics & $0.0056(1)$ \\
$\alpha K_{T}(\mathrm{GPa} / \mathrm{K})$ & $-0.0042(2)$ \\
$\left(\partial K_{T} / \partial T\right)_{V}(\mathrm{GPa} / \mathrm{K})$ & $6.0(26)$ \\
$\left(\partial^{2} K_{T} / \partial T^{2}\right)_{V}\left(10-{ }^{8} \mathrm{GPa}^{2} / \mathrm{K}^{2}\right)$ & \\
\hline
\end{tabular}


TABle 2: Comparison of the elastic parameters of Re at room temperature.

\begin{tabular}{lccc}
\hline$K_{T 0}(\mathrm{GPa})$ & $K_{T}^{\prime}$ & $\mathrm{V}_{0}\left(\mathrm{~cm}^{3} / \mathrm{mol}\right)$ & \\
\hline $\begin{array}{l}\text { Experiments } \\
336\end{array}$ & 4 & & X-ray diffraction [2] \\
360.3 & 5.43 & & Ultrasonic [5] \\
353 & 5.80 & 8.87 & X-ray diffraction [3] \\
352.6 & 4.56 & 8.8726 & X-ray diffraction [4] \\
383 & 3.26 & 8.878 & X-ray diffraction, present study \\
$\begin{array}{lcc}\text { Calculations } \\
447\end{array}$ & & LDA [6] \\
382 & 3.9 & 8.764 & LDA [7] \\
344 & 3.9 & 9.192 & GGA [7] \\
389 & 4.52 & 8.789 & LDA [8] \\
376 & 4.58 & 8.842 & GGA [8]
\end{tabular}

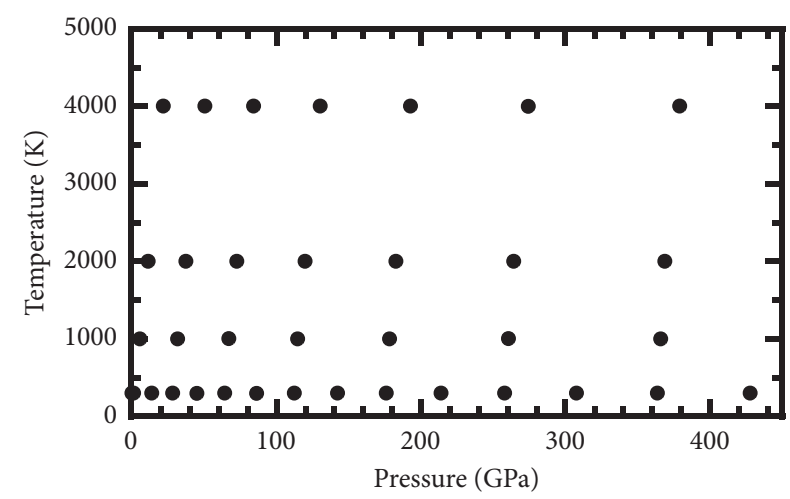

Figure 3: Pressure-temperature conditions under which firstprinciples molecular dynamics was performed.

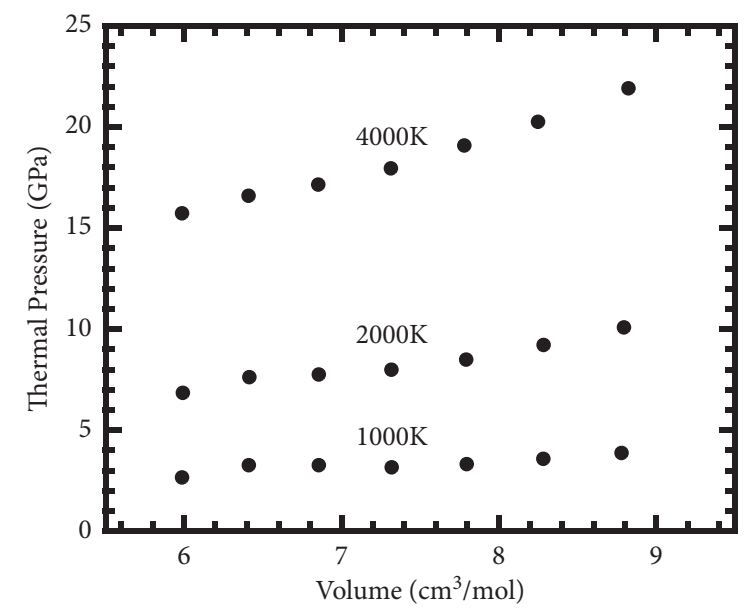

FIGURE 4: Plot of the thermal pressure calculated using firstprinciples molecular dynamics. Solid circles represent the calculated thermal pressures at 1000,2000 , and $4000 \mathrm{~K}$.

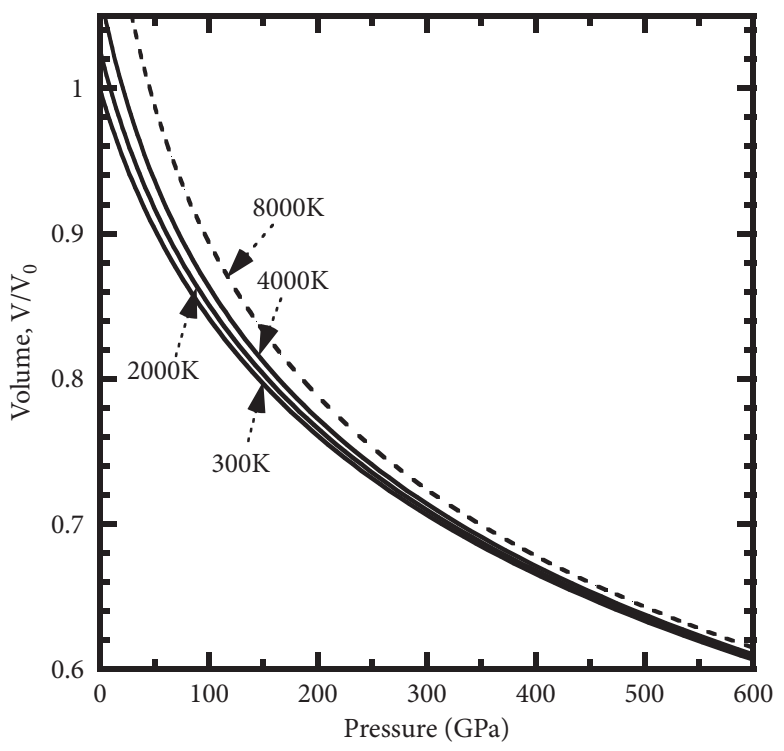

FIgURE 5: Change in the isothermal volume of Re. Solid lines are the calculated isotherms at 300,2000 , and $4000 \mathrm{~K}$ using equation (4) and the parameters in Table 1. Dashed line represents the explored isotherm at $8000 \mathrm{~K}$.

\section{Data Availability}

The data supporting the findings of this study are available upon request to the author.

\section{Conflicts of Interest}

The author declares that there are no conflicts of interest.

\section{Acknowledgments}

This work made use of the DA system of JAMSTEC. The synchrotron radiation experiments were performed at the PF, KEK (Proposal No. 2019G503) and at the SPring-8, JASRI (Proposal No. 2019A1099). The author thanks T. Kikegawa, N. Hirao, and Y. Ohishi for their experimental help. This work was partially supported by JSPS KAKENHI (Grant no. JP18K03792).

\section{References}

[1] P. W. Bridgemann, Proceedings of the American Academy of Arts and Sciences, MIT Press, vol. 84, p. 111, Cambridge, MA, USA, 1955.

[2] L.-G. Liu, T. Takahashi, and W. A. Bassett, "Effect of pressure and temperature on the lattice parameters of rhenium," Journal of Physics and Chemistry of Solids, vol. 31, no. 6, pp. 1345-1351, 1970.

[3] L. Dubrovinsky, N. Dubrovinskaia, V. B. Prakapenka, and A. M. Abakumov, "Implementation of micro-ball 
nanodiamond anvils for high-pressure studies above 6 Mbar," Nature Communications, vol. 3, no. 1, p. 1163, 2012.

[4] S. Anzellini, A. Dewaele, F. Occelli, P. Loubeyre, and M. Mezouar, "Equation of state of rhenium and application for ultra high pressure calibration," Journal of Applied Physics, vol. 115, no. 4, Article ID 043511, 2014.

[5] M. H. Manghnani, K. Katahara, and E. S. Fisher, "Ultrasonic equation of state of rhenium," Physical Review B, vol. 9, no. 4, pp. 1421-1431, 1974.

[6] L. Fast, J. M. Wills, B. Johansson, and O. Eriksson, "Elastic constants of hexagonal transition metals: Theory," Physical Review B, vol. 51, no. 24, pp. 17431-17438, 1995.

[7] G. Steinle-Neumann, L. Stixrude, and R. E. Cohen, "Firstprinciples elastic constants for the hcp transition metals $\mathrm{Fe}$, Co, and Re at high pressure," Physical Review B, vol. 60, no. 2, pp. 791-799, 1999.

[8] M.-B. Lv, Y. Cheng, Y.-Y. Qi, G.-F. Ji, and C.-G. Piao, "Elastic properties and phonon dispersions of rhenium in hexagonalclose-packed structure under pressure from first principles," Physica B: Condensed Matter, vol. 407, no. 4, pp. 778-783, 2012.

[9] N. Dubrovinskaia, L. Dubrovinsky, N. A. Sollopova et al., "Terapascal static pressure generation with ultrahigh yield strength nanodiamond," Science Advances, vol. 2, Article ID e1600341, 2016

[10] T. Sakai, T. Yagi, T. Irifune et al., "High pressure generation using double-stage diamond anvil technique: problems and equations of state of rhenium," High Pressure Research, vol. 38, no. 2, pp. 107-119, 2018.

[11] A. Dewaele, P. Loubeyre, F. Occelli, O. Marie, and M. Mezouar, "Toroidal diamond anvil cell for detailed measurements under extreme static pressures," Nature Communications, vol. 9, no. 1, p. 2913, 2018.

[12] G. Kresse and J. Furthmüller, "Efficient iterative schemes forab initiototal-energy calculations using a plane-wave basis set," Physical Review B, vol. 54, no. 16, pp. 11169-11186, 1996.

[13] J. P. Perdew, A. Ruzsinszky, G. I. Csonka et al., "Restoring the density-gradient expansion for exchange in solids and surfaces," Physical Review Letters, vol. 100, no. 13, Article ID 136406, 2008.

[14] S. Nosé, "A molecular dynamics method for simulations in the canonical ensemble," Molecular Physics, vol. 52, p. 255, 1984.

[15] S. Ono, T. Kikegawa, and Y. Ohishi, "A high-pressure and high-temperature synthesis of platinum carbide," Solid State Communications, vol. 133, no. 1, pp. 55-59, 2005.

[16] S. Ono, Y. Ohishi, and T. Kikegawa, "High-pressure study of rhombohedral iron oxide, $\mathrm{FeO}$, at pressures between 41 and 142 GPa," Journal of Physics: Condensed Matter, vol. 19, no. 3, Article ID 036205, 2007.

[17] S. Ono, K. Funakoshi, Y. Ohishi, and E. Takahashi, "In situxray observation of the phase transformation of $\mathrm{Fe} 2 \mathrm{O} 3$," Journal of Physics: Condensed Matter, vol. 17, no. 2, pp. 269-276, 2005.

[18] S. Ono and T. Kikegawa, "Determination of the phase boundary of GaP using in situ high pressure and high-temperature X-ray diffraction," High Pressure Research, vol. 37, no. 1, pp. 28-35, 2017.

[19] P. I. Dorogokupets and A. Dewaele, "Equations of state of $\mathrm{MgO}, \mathrm{Au}, \mathrm{Pt}, \mathrm{NaCl}-\mathrm{B} 1$, and $\mathrm{NaCl}-\mathrm{B} 2$ : internally consistent high-temperature pressure scales," High Pressure Research, vol. 27, no. 4, pp. 431-446, 2007.

[20] P. V. J. Ferrante, J. Rose, and J. Smith, "Compressibility of solids,” Journal of Geophysical Research, vol. 92, pp. 9319-9325, 1987.
[21] S. Ono, K. Mibe, and Y. Ohishi, "Raman spectra of culet face of diamond anvils and application as optical pressure sensor to high temperatures," Journal of Applied Physics, vol. 116, no. 5, Article ID 053517, 2014.

[22] I. Jackson and S. M. Rigden, "Analysis of P-V-T data: constraints on the thermoelastic properties of high-pressure minerals," Physics of the Earth and Planetary Interiors, vol. 96, no. 2-3, pp. 85-112, 1996.

[23] S. Ono, "First-principles molecular dynamics calculations of the equation of state for tantalum," International Journal of Molecular Sciences, vol. 10, no. 10, pp. 4342-4351, 2009.

[24] S. Ono, "The equation of state of B2-type NaCl," Journal of Physics: Conference Series, vol. 215, Article ID 012196, 2010.

[25] S. Ono, "Fate of subducted argon in the deep mantle," Scientific Reports, vol. 10, no. 1, p. 1393, 2020.

[26] Y. K. Vohra, S. J. Duclos, and A. L. Ruoff, "High-pressure $\mathrm{x}$-ray diffraction studies on rhenium up to $216 \mathrm{GPa}(2.16$ Mbar)," Physical Review B, vol. 36, no. 18, pp. 9790-9792, 1987.

[27] C. S. Perreault, N. Velisavljevic, and Y. K. Vohra, "Highpressure structural parameters and equation of state of osmium to 207 GPa," Cogent Physics, vol. 4, no. 1, p. 1376899 , 2017.

[28] W. J. Evans, M. J. Lipp, H. Cynn et al., Physical Review B: Condensed Matter, vol. 72, Article ID 09413, 2005.

[29] R. Torchio, C. Marini, Y. O. Kvashnin et al., Physical Review B: Condensed Matter, vol. 94, Article ID 02429, 2016.

[30] O. Tschauner, O. Grubor-Urosevic, P. Dera, and S. R. Mulcahy, "Anomalous elastic behavior in hcp- and Smtype dysprosium," Journal of Physical Chemistry C, vol. 116, no. 3, pp. 2090-2096, 2011.

[31] A. K. Verma, P. Ravindran, R. S. Rao, B. K. Godwal, and R. Jeanloz, "On the stability of rhenium up to $1 \mathrm{TPa}$ pressure against transition to thebcc structure," Bulletin of Materials Science, vol. 26, no. 1, pp. 183-187, 2003.

[32] G. L. Rech, J. E. Zorzi, and C. A. Perottoni, "Equation of state of hexagonal-close-packed rhenium in the terapascal regime," Physical Review B, vol. 100, no. 17, p. 174107, 2019. 